\title{
Charles Percier et les bandeaux des Fables
}

\section{Bernard Teyssandier}

\section{Citer ce document / Cite this document :}

Teyssandier Bernard. Charles Percier et les bandeaux des Fables. In: Le Fablier. Revue des Amis de Jean de La Fontaine, $n^{\circ} 25$, 2014. La Fontaine, la fable et l'image. Actes du colloque international 6 \& 7 décembre 2012. Deuxième partie. pp. 47-53; doi : https://doi.org/10.3406/lefab.2014.1243

https://www.persee.fr/doc/lefab_0996-6560_2014_num_25_1_1243

Fichier pdf généré le 09/03/2020 


\section{CHARLES PERCIER ET LES BANDEAUX DES FABLES}

Les vignettes de François Chauveau (1613-1676) qui, en 1668, ornent l'édition Barbin constituent le décalque analogique non pas tant des fables ellesmêmes que de leurs titres'. Tout en intégrant le décor à l'histoire, le graveur dispose au premier plan les figurants à partir desquels s'organise le récit lafontainien : l'enfant et le maître d'école « sur les bords de la Seine », le coq et la perle dans une basse11 cour, le «père » des fables expliquant son testament aux trois filles « dans la ville d'Athènes », etc. Ce faisant, Chauveau s'inscrit dans une tradition iconographique qu'on dira par commodité "s ésopique ", tradition qui le précède et qui lui survit très largement. En 1755, mutatis mutandis, c'est encore le même procédé qui est adopté pour l'édition en quatre volumes parue chez Desaint et Saillant à partir de dessins originaux de Jean-Baptiste Oudry $^{2}$ (1686-1755) : les titres présentent les acteurs, et les images les représentent, tandis que l'apologue déroule le fil d'une narration qui inscrit l'histoire dans une temporalité dramatique ${ }^{3}$.

Changement radical en 1802 avec la parution en deux volumes in-folio chez Pierre Didot l'aîné (1761-1853) d'une nouvelle édition des Fables : l'architecte Charles Percier (1764-1838) n'illustre plus les apologues de La Fontaine à proprement parler, il couronne chacun des douze livres du recueil d'un bandeau liminaire. Près de cinquante ans après les gravures réalisées d'après des dessins d'Oudry, cette édition bientôt dite " du Louvre » enregistre un glissement majeur par rapport aux normes éditoriales régissant la distribution de la fable et de l'image. Comprendre la logique qui préside à cette évolution, voire à cette révolution, formuler des hypothèses pouvant la justifier et l'interpréter, tel sera l'objet de cette étude.

En 1797, le gouvernement révolutionnaire confie à Pierre Didot les salles de l'Imprimerie royale situées dans un des bâtiments du Louvre. Ce sont dans ces lieux de mémoire que la collection "du Louvre ", ainsi nommée du fait qu'elle avait été imprimée dans l'enceinte du célèbre palais, voit le jour'. Pour mener à bien son projet, Pierre Didot s'inspire des premières grandes réalisations de l'Imprimerie royale, celles des années $1640^{5}$. Il perpétue ainsi la tradition du livre d'apparat - format in-folio, grandes marges, grand papier, gravures sur cuivre, ornementations dédiées ${ }^{6}$. En n'éditant que quelques rares auteurs, il renoue aussi avec la politique de prestige de cette institution d'État ${ }^{7}$. Comme Sébastien Cramoisy (1585-1669), à qui avait

(1) Alain-Marie Bassy, « Du texte à l'illustration : pour une sémiologie des étapes ", Semiotica, n 11 , 1974, p. 303.

(2) L'édition Desaint et Saillant (1755-1759) paraît en quatre volumes in-folio : les 276 figures à pleines pages constituent de véritables tableaux (210 $\mathrm{mm}$ de largeur pour 270 de hauteur $)$ et sont pour la plupart gravées au burin d'après des dessins d'Oudry. L'édition pratique parfois la "duplication " de la gravure, à deux reprises l'image est même triplée. Voir Jean-Pierre Collinet, "La Fontaine et ses illustrateurs ", dans son édition des Euvres complètes de La Fontaine, Paris, Gallimard, «Bibliothèque de la Pléiade », 1991, 2 vol., t. I, p. Lxx-1.xxxvit.

(3) Patrick Dandrey, "Le talent qu'il avait de conter" : l'art de la narration dans les Fables de La Fontaine ", Le Fablier, n² 23, 2012, p. 13-20.

(4) Pour promouvoir leur production imprimée, Pierre Didot et son frère Firmin publièrent des Prospectus. Le document papier, joint à l'exemplaire BnF des CEuvres de Virgile dans l'édition « du Louvre » de 1798 [Res-a:las-YC-1], fait état d'une gestation de sept ans pour la parution du premier volume de la collection.

(5) Ces éditions sont notamment marquées par la collaboration de Nicolas Poussin (1594-1665) et de Claude Mellan (1598-1688) : Publii Virgilii Maronis, Opera, Paris, e Typographia Regia, 1641 ; Biblia sacra latina, Paris, e Typographia Regia, 1642 ; Quinti Horatii Flacci, Opera, Paris, e Typographia Regia, 1642. Voir Carol Margot Osborne, Pierre Didot the Elder and French Book Illustration 17891822, New York et Londres, Garland Publishing, 1985, p. 61.

(6) Jean-Marc Chatelain, «Pour la gloire de Dieu et du roi : le livre de prestige au XVII" siècle ", dans Henri-Jean Martin, La Naissance du livre moderne (XII -XVIT siècle) : mise en page et mise en texte du livre français, Paris, Éditions du Cercle de la Librairie, 2000, p. 350363 ; "Formes et enjeux de l'illustration du livre au XVII" siècle : le livere d'apparat ". Cahiers del'Association Internationale des litudes Françaises, n 57, 2005, p. 75-98.

(7) Auguste Bernard, Histoire de /\%mprimerie rolale du Lonwe, Paris. Imprimerie impériale, 1867 ; Paul-Marie Grinevald, "Richelieu et I'Imprimeric royale ", dans Richelieu et le monde de l'esprit, Paris, Imprimerie nationale, 1985,p. 237-248; Jacques Thuillier, « Richelieu et les arts. L'Imprimerie royale ", dans Roland Mousnier (dir.), Richelieu et la culture. Actes du colloque international en Sorbonne (19-20 novembre 1985), Paris, Éditions du CNRS, 1987, p. 163-174. 
été confiée "la direction technique de l'entreprise ${ }^{8}$ ", Didot célèbre les grands textes de la littérature latine : paraissent successivement les Euvres de Virgile (1798) et celles d'Horace (1799)'. Marchant sur les pas de Richelieu et de Sublet des Noyers, mais aussi de Malherbe et de Vaugelas, il souscrit à l'hypothèse d'une stabilité rhétorique et historique du français garantie par « un jeu institutionnel souple, évolutif, mais fidèle à un certain nombre de "règles" qui guident en sûreté le "bon" et "bel" usage ${ }^{10} \gg$. Le fait de publier quelquesuns des grands chefs-d'œuvre du siècle de Louis le Grand le conduit ainsi, après bien d'autres, à défendre l'idée d'une passation, d'une translatio" ${ }^{\text {", conférant à }}$ la nation France la gloire des puissances immortelles ${ }^{12}$. Un an avant les Fables de La Fontaine, le premier des trois volumes du théâtre de Racine est imprimé. Le dernier titre de la collection dite « du Louvre " paraît en 1819 avec les Euvres de Boileau en deux volumes ${ }^{13}$.

Or ce projet monumental est pour Didot l'occasion d'assurer la promotion de sa propre famille ; l'éditeur parisien, lui-même fils et petit-fils d'éditeurs, ne se contente pas d'apparaître comme un héritier ni même comme un passeur : la " société des cinq auteurs " (Virgile, Horace, Racine, La Fontaine et Boileau) qu'il réunit sous son nom lui permet d'affirmer l'existence et même l'efficience d'une « dynastie Didot ». En 1784 avait déjà paru sous son nom une Épitre sur les progrès de l'imprimerie, texte manifeste sous-tendu par l'idée que la pratique éditoriale ne pouvait plus se penser en dehors d'une conscience historique d'ellemêmc. Dans cet opusculc, Picrre Didot soutenait que l'art typographique était parvenu à un tel degré de modernisation qu'il devait envisager sa propre fin. L'ouvrage prétendait démontrer que la génération de François Didot (1689-1757) le grand-père, et surtout celle de François-Ambroise (1730-1804) le père ${ }^{14}$, avaient hissé l'imprimerie à une telle hauteur que dorénavant elle était indépassable. Or c'est à nouveau la perspective d'un absolu typographique qui est défendue en 1798 au moment de la parution des Euvres de Virgile : le Rapport sur le Virgile de Didot l'aîné publié chez l'Imprimeur de l'Institut national et rédigé par le « citoyen Camus au nom d'unc commission spéciale " entérine l'idée d'une ambition au service d'une triple perfection : perfection philologique dans le choix et l'établissement des textes, perfection dans la fonte des caractères et dans la mise en page, perfection dans la réalisation plastique des figures gravées. La collection « du Louvre » se présente ainsi comme le résultat de plusieurs décennies de recherches et d'expériences, qu'il s'agisse de l'invention des fameux caractères « Didot » spécialement conçus par le frère de Pierre, Firmin (1764-1836), du papier vélin à grandes marges et à la blancheur uniforme et impeccable ${ }^{15}$, de l'encre d'un noir profond à même de réaliser les plus parfaits contrastes, des effets graphiques destinés à promouvoir l'idée d'autorité, des corrections scrupuleusement accomplies ou encore de l'insertion de gravures inédites conçues pour accompagner les textes ${ }^{16}$.

Dans une communication récente ${ }^{17}$, Jean-Marc
Chatelain et moi-même avions soutenu l'idée selon laquelle l'esthétique du sublime avait inspiré le projet de Didot. Nous avions évoqué, à propos des productions " du Louvre », un imaginaire de l'extension et de l'intensité, un goût du lisse, goût néo-classique dans l'utilisation du blanc notamment, dont Mario Praz, citant Winckelmann, considérait qu'il avait vocation à représenter la grandeur et l'éclat ${ }^{18}$. Cette collection qui, en fin de compte, se solda par un échec financier, était sans doute moins destinée à être lue qu'à être vue. Devant de tels ouvrages (les pages comptent près de $50 \mathrm{~cm}$ de hauteur pour $35 \mathrm{~cm}$ de largeur), le supposé lecteur est soumis à une présence qui exclut tout phénomène d'identification. Entre elle et le public s'instaure une distance faite d'admiration contemplative et de respect contenu. La « grande » littérature, la seule qui, selon

(8) H.-J. Martin, Livre, pouvoirs et société à Paris au XVIr siècle, Genève, Dro7., 1999, 2 vol., t. I, p. 468.

(9) Publius Virgilius Maro, Bucolica, Georgica et Eneis, Paris, in ædibus Palatinus, 1798, excudebat Petrus Didot, natu maior ; Quintus Horatius Flaccus, Opera, Paris, in adibus Palatinus, excudebam Petrus Didot, natu maior, 1799. Mellan travaille d'après des dessins de Jacques Stella pour les éditions de l'Introdaction à la vie dévote (1640), du De Imitatione Christi (1640), des (Euvres de saint Bernard (1642). Sur l'Imprimerie royale, voir H.-J. Martin, Live, pouvoirs et société à Paris au XVII' siècle, op. cit., t. I, p. 467 sqq.

(10) Marc Fumaroli, " "Le génie de la langue française" ", dans Trois institutions littéraires, Paris, Gallimard, 1994, p. 274.

(11) E. R. Curtius, La Littérature européenne et le Moven Age latin, trad. Jean Bréjoux, Paris, PUF, 1956, p. 71.

(12) Sur la « vocation » de la langue française, langue du roi et du royaume, à exercer l'empire du savoir et du pouvoir, voir M. Fumaroli, art. cit., p. 224 sqq.

(13) Euvres de Jean Racine, Paris, imprimerie de Pierre Didot l'aîné, 1801-1805, 3 vol. ; Fables de La Fontaine, Paris, de l'imprimerie de Pierre Didot l'aîné, Au Palais national des Sciences et Arts, 1802, 2 vol. ; Fuvres de Boileau, édition dédiée au roi, Paris, de l'imprimerie et de la fonderie de Pierre Didot l'aîné, chevalier de l'ordre royal de Saint-Michel, Imprimeur du roi et de la Chambre des pairs, et Jules Didot fils, chevalier de la Légion d'honneur, 1819, 2 vol. Le volume in-folio consacré aux cuvres de Salluste, paru en 1819 (C. Crispi Sallustii Catilinaria et Jugurthina bella), publié sous le nom de Firmin Didot et non de Pierre, ne présente ni illustration ni ornementation, ce qui autorise à ne pas l'associer à la collection des " éditions du Louvre ».

(14) François-Ambroise Didot fut à l'origine d'une nouvelle normalisation de la taille des caractères d'imprimerie. Il fit graver par Wafflard des caractères spécifiques bientôt appelés « Didot»; il introduisit aussi le papier vélin ainsi que la presse dite «à un coup », ce qui permit d'obtenir une surface d'impression plus égale. Voir Les Didot. Trois siècles de typographie et de bibliophilie 1698-1998, catalogue par André Jammes, Paris, Agence culturelle de Paris, 1998.

(15) Didot revient notamment sur ce point dans les « Notes » qui suivent son Épitre sur les progrès de l'imprimerie [1784], Paris, J. Haumont, 1941, p. 125.

(16) «Les Didot n'ignorent pas, vous les avez. vus le déclarer, qu'on peut avancer plus près du beau parfait dont ils se forment l'idée ", dans Rapport sur le Virgile de Didot l'aîné, Baudoin, Imprimeur de I'Institut national, an VI, p. 10.

(17) «Typographie et scénographie du livre néo-classique : l'ambition de Pierre Didot n, communication prononcée lors du colloque de Reims, "Visages du livre : l'iconographic de la page de titre ", 13-15 mars 2008, à paraître.

(18) «Comme la blancheur est, de toutes les couleurs, celle qui réfléchit le plus de rayons et par conséquent frappe plus sensiblement l'œil, il en résulte que la blancheur augmente la beauté d'un beau corps : regardé nu, il paraitra plus grand qu'il ne l'est en effet ", Winckelmann, cité par Mario Praz, dans Gouit néo-classique, trad. Constance Thompson Pasquali. Paris, Le Promeneur, 1989, p. 85. 
Didot, mérite de durer, est à la fois intransitive (elle ne sollicite pas l'échange), et politique (elle est au service d'un ordre). Sa légitimité procède du sentiment de puissance qui en émane ${ }^{19}$.

D'esprit burlesque et de veine parfois satirique, les Fables de La Fontaine que Boileau néglige superbement dans son Art poétique (1674) n'avaient pas vocation à rejoindre un tel projet ${ }^{20}$. Autant dire qu'en agrégeant ces textes facétieux à un ouvrage dont l'ambition, pour reprendre les mots de Boileau, est d'enlever, de ravir et de transporter ${ }^{21}$, Didot joue les apprentis sorciers. La première inflexion qu'il imprime ou qu'il inflige à l'œuvre de La Fontaine, tient à l'établissement du texte. S'il conserve la structure initiale d'un recueil divisé en douze livres, il exclut très symboliquement du dernier massif dédié au duc de Bourgogne deux histoires galantes dont la légèreté badine et libertine consonne mal avec l'esprit de sa collection, celle de la Matrone d'Éphèse, d'inspiration licencieuse (Pétrone), et celle de Belphégor, libre adaptation d'après Machiavel. Dans le même temps, Didot reproduit toutes les pièces liminaires du recueil de 1668 , chacune d'entre elles constituant un plaidoyer en faveur des vertus civilisatrices et éducatives de l'apologue au-delà même de leur dimension encomiastique ${ }^{22}$. Deuxième inflexion, qui concerne la mise en page des fables cette fois-ci. Didot relie en fil continu les textes dissociés, ce qui confère à l'ensemble une certaine ampleur : la distribution des fables en livres, plus que jamais, se fait par blocs. Les historiettes s'enchaînent les unes aux autres, séparées par un simple trait horizontal, aucune d'entre elles, aussi courte soit-elle, ne venant interrompre le rythme et même la cadence de l'inscription typographique. Aussi le vide qui marque la fin de chacun des douze livres du recueil interromptil moins le continuum narratif qu'il n'annonce une apparition : celle, rare, et de fait remarquable, de l'image.

Or, malgré leurs dimensions modestes (près de $17 \mathrm{~cm}$ de large pour $6 \mathrm{~cm}$ de hauteur), les dessins de Percier $^{23}$ confortent le plus souvent les choix éditoriaux de Didot. Leur finalité est triple en réalité. Pcrcicr s'ingćnic d'abord à rendre la fable grande en l'historicisant. Deux époques mythiques sont privilégiées. Celle de l'Antiquité au premier chef. Pour "La Laitière et le Pot au lait " par exemple, c'est à Rome que se déroule l'histoire alors même que le poète ne donne aucune indication de lieu. Or, si Percier habille sa servante d'un « costume provincial français de son époque $^{24}$ ", il représente un arc de triomphe en point de fuite afin de magnifier l'anecdote. Même procédé d'incrustation avec le bandeau du livre XI illustrant "Le Paysan du Danube " : la sculpture de Remus et Romulus nourris par la Louve accuse la dimension universelle d'une scène dont la représentation en frise (adoptée aussi pour le bandeau du livre IX) souligne le caractère théâtral. La seconde époque que Percier choisit de mettre en valeur, tout aussi célèbre que la précédente, est celle de la Renaissance ${ }^{25}$, et plus encore de la Renaissance française : fenêtres à meneaux dans la vaste salle réunissant " Le Vieillard et ses Enfants » (livre IV), culottes Henri III et manches à crevés pour le maître d'école (livre I), pour les protagonistes du «Berger et le Roi » (livre X), pour l'astrologue (livre II), pour les fils du vieillard enfin (livre IV) ${ }^{26}$. Ces scènes

(19) Paul Veyne, "Propagande expression roi, image idole oracle ", dans Id. et Louis Marin, Propagande expression roi, image idole oracle. Visibilité et lisibilité des images du pouvoir, Paris, Arkhê, " Figurer ", 2011, p. 13-46, notamment p. 41-44. André Chastcl, L'Art français. Le temps de l'éloquence 1775-1825, Paris, Flammarion, 4 vol., t. IV, 1996, p. 27-28. A.-M. Bassy, "Le texte et l'image ", dans H.-J. Martin et Roger Chartier (dir.), Histoire de l'édition française. Le live triomphant 1660-1830 [1984], Paris, Fayard, 1990, p. 181. Du même auteur, voir « Du texte à l'illustration : pour une sémiologie des étapes », art. cit., p. 334 : l'auteur analyse le bandeau du livre III de Percier tout en soulignant la dimension idéologique de l'image.

(20) Non pas que le burlesque, évidemment, ait fait l'objet d'une quelconque désapprobation dans la société de cour du XVII' siècle. Dès l'année qui suit la publication de L'Art poétique de Boileau, La Fontaine est associé à la création de la «Chambre du sublime » offerte en étrennes au duc du Maine par Madame de Thianges. Boileau est d'ailleurs luimême représenté en figurine non loin du fabuliste. Mais à l'orée du $\mathrm{XIX}^{*}$ siècle. Pierre Didot se situe dans une autre perspective pour son édition des Fables: son esthétique n'est plus ni mondaine ni galante, aussi la dimension de radicalité du traité de Boileau lui permet-clle de redéfinir l'univers des fables et d'imprimer sa propre lecture des apologues lafontainiens. Nous renvoyons sur ce point aux conclusions du présent article.

(21) On reconnaît ici les catégories proposées par Boileau pour définir le style sublime : voir Francis Goyet (èd.), Longin. Traité du sublime. Traduction de Boilecu, Paris, Librairie Générale Française, "Le Livre de poche (713) », 1995, p. 70.

(22) Épître dédicatoire « À Monseigneur le Dauphin »: «S’il y a quelque chose d'ingénicux dans la république des Lettres, on peut dire que c'est la manière dont Ésope a débitć sa morale. [...] L'apparence [des fables que nous devons à Ésope] en est puérile, je le confesse ; mais ces puérilités servent d'enveloppe à des vérités importantes. [...] La lecture de son ouvrage répand insensiblement dans une âme les semences de la vertu, et lui apprend à se connaître sans qu'elle s'aperçoive de cette étude, et tandis qu'elle croit faire toute autre chose "; "Préface »: "À peine les fables qu'on attribue à Ésope virent le jour, que Socrate trouva à propos de les habiller des livrées des Muses [...] La vérité a parlé aux hommes par paraboles; et la parabole est-elle autre chose que l'apologue ? C"est pour cette raison que Platon ayant banni Homère de sa république, y a donné à Ésope une place très honorable. [...] Ces badineries ne sont telles qu'en apparence; car dans le fond elles portent un sens très solide. [...] Par les raisonnements et conséquences que l'on peut tirer de ces fables, on se forme le jugement et les mœurs, on se rend capable de grandes choses. Elles ne sont pas seulement morales, elles donnent encore d'autres connaissances. [...] Ainsi ces fables sont un tableau où chacun de nous se trouve dépeint "; " La Vie d'Ésope le Phrygien »: «Quant à Ésope, il me scmble qu'on le devrait mettre au nombre des sages dont la Grèce s'est vantée, lui qui enseignait la véritable sagesse, et qui l'enseignait avec bien plus d'art que ceux qui en donnent des définitions et des règles " ; Épître en vers «À Monseigneur le Dauphin »: « Je chante les héros dont Ésope est le père, / Troupe de qui l'histoire encor que mensongère, / Contient des vérités qui servent de leçons.[...] Je me sers d'animaux pour instruire les hommes ».

(23) Douze « gravures-frontispices » en taille-douce gravées par sept burinistes.

(24) A.-M. Bassy, Les Fables de La Fontaine. Quatre siècles d'illustration, Paris, Promodis, 1986, p. 197

(25) "Les intérieurs de Percier sont tous des intéricurs de la Renaissance. Voyez ee splendide cabinel d" astronomic de lastrologuc qui se laisse tomber dans un puits. Son porche s'ouvre sur deux pilastres marqués aux signes du zodiaque et reposant sur des lions de style lombard. Devant une baie à double ogive et rosace, dont les carreaux losangés diffusent une lumière tamisée, on aperçoit la lourde table de travail à pieds torsadés, sur laquelle reposent des instruments de travail de l'astrologue : mappemonde, sablier, niveau », ibid., p. 184.

(26) «L'on peut se demander pourquoi les costumes des derniers Valois furent préfërés à d’autres vêtements moins singuliers. La réponse 
rétrospectives qui s'inspirent très largement du « style troubadour ${ }^{27} \gg$, tant pour ce qui est du luxe des costumes que du faste des décors, contribuent à solenniser l'ouvrage. Percier proscrit d'ailleurs tout prosaïsme dans ses compositions : l'esprit " silénique ${ }^{28}$ " de la tradition ésopique est systématiquement embelli, l'ornementation triomphe (figure 1).

Percier s'emploie ensuite à sophistiquer la fable. Contrairement à Gérard et Girodet, ou encore à Prud'hon, qui recourent aux figures à pleines pages pour l'illustration du Virgile et du Racine, il adopte le bandeau, fidèle en cela au choix qui fut le sien pour les CEuvres d'Horace. Mais ce que l'artiste perd en surface, il le gagne en profondeur. Il ne s'agit plus tant en effet d'agréer le public en le soumettant à l'illusion d'une « peinture » que de l'installer dans une modalité d'appréhension du sens qui ne fonctionne plus seulement sur le mode conventionnel d'une lecture par plans successifs (premier plan, second plan), mais par " morcellement itératif ${ }^{29}$ ". Le bandeau du livre VI est à ce titre révélateur (figure 2). À la forme rectangulaire vient s'agréger celle, moins attendue, du cercle. Mais rien de fortuit dans ce choix, bien entendu. Percier s'inspire en réalité de l'art numismatique conformément à l'inflexion antiquisante que Didot imprime à sa collection. En réponse à la fable de La Fontaine organisée en deux séquences distinctes et complémentaires, il imagine un dispositif double, avec son avers et son revers, avec son avant et son après. Des parcours d'observations originaux sont ainsi suggérés : l'image se lit encore, certes, comme un texte, de gauche à droite, mais elle s'offre aussi au regard en hauteur, en profondeur et même parfois de manière transversale. Le lieu de l'image devient ainsi le lieu d'expériences visuelles et d'observations optiques raffinées ${ }^{30}$. Le cercle de moindre dimension qui relie les deux médailles du bandeau du livre $\mathrm{VI}^{31}$ évoque à la fois l'anneau du mariage, les signes du zodiaque et la roue de Fortune. Quant au miroir dans lequel se mire la veuve ragaillardie, il dessine, sans que la malheureuse y prenne garde, le visage émacié d'une vieille décrépite. La gravure fait ainsi discrètement écho à « L'Homme et son image ", à moins qu'elle n'affirme sur le mode mineur la dimension spéculaire du recueil tout entier. L'idée d'une lecture globale et croisée de l'œuvre est d'ailleurs suggérée par Percier lui-même : sur les douze bandeaux qu'il compose, six d'entre eux illustrent non pas une seule mais plusieurs fables ${ }^{32}$.

Le "très aristocratique Percier $^{33}$ " s'attache enfin à rendre la fable belle en l'esthétisant. Un imaginaire architectural est à l'origine de l'ensemble de ces figures : succession de blocs formant entablements, piédestaux, marches, effet d'élévation par insertions multiples de colonnes, goût pour les bustes sculptés plus ou moins discrètement disposés. Les objets gagnent d'ailleurs en visibilité dans l'édition de 1802 , soit parce qu'ils figurent sur les panneaux centraux, prenant directement part à l'action au même titre que les humains, soit parce qu'ils ornent les décors latéraux au titre d'attributs symboliques. Quant aux animaux, à la fois stylisés et réifiés, ils sont le plus souvent réduits à l'état de miniatures, exposés aux regards des spectateurs tels des trophées. Cette "sublimation plastique ${ }^{34}$ ", qui s'inscrit dans une réalité d'époque, les fables illustrant parfois les boiseries des salles à manger, traduit une forme d'idéalisation de l'apologue. Percier, on l'a noté, proscrit tout effet de prosaïsme, mais il va plus loin : animaux et objets prennent « la forme figurée de concepts » et se constituent en " garants de la fable humaine ${ }^{35}$ ", voire de la fable urbaine.

Dans l'ample recueil de La Fontaine, Percier opère ainsi une sélection qui ne doit rien au hasard. Soit la fable qu'il choisit rassemble elle-même de nombreuses formules gnomiques, ce qui est le cas par exemple pour " La Laitière et le Pot au lait ", soit elle aborde de grandes questions morales : l'amitié, la prudence, la justice, le passage de la vie à la mort (avec la scène du " lit funéraire ${ }^{36}$ " au livre IV), l'idée d'éducation enfin ${ }^{37}$. Didot, rappelons-lc, public les deux volumes consacrés à La Fontaine en 1802, époque propice à la commémoration mais aussi à la refondation politique ${ }^{38}$. Or les gravures de Percier, justement, ont pour principal objectif de valoriser l'autorité de l'exemple. Dans ces conditions " l'esprit " des Fables devient secondaire. Pour le bandeau du livre XII, par exemple, c'est moins l'anecdote de l'Amour aveuglé par la Folie

est dans l'oubli de ce qui avait existé avant la Renaissance et dans un décalage chronologique suffisant pour donner le cachet de l'anciennete aux modes d'antan ", François Pupil, Le Stvle troubadou'. ou la nostalgie du bon vieux temps, Nancy, Presses Universitaires de Nancy, 1985, p. 251.

(27) F. Pupil insiste sur la dimension politique de ces « entreprises rétrospectives " qui voicnt le jour dès le XVII" siècle : ibid., p. 190 et p. 383. L'auteur date néanmoins la naissance du style et de la fantaisie « troubadour » des années 1790.

(28) B. Teyssandier, "Postface ", dans Jean Ballesdens, Les Fables d'Ésope Phryien, Rcims, Épure, 2011, p. 490.

(29) A.-M. Bassy, « Du texte à l'illustration : pour une sćmiologie des étapes ", art. cit., p. 324.

(30) Effet d'écho en forme d'hommage : la représentation du « Renard et la Cigogne » sur les pans latéraux du bandeau du livre I est clairement un calque de l'illustration réalisée pour l'édition de 1755 parue chez Desaint et Saillant.

(31) A.-M. Bassy, Les Fables de La Fontaine. Quatre siècles d'illustration, op. cit., p. 83 .

(32) Livre I (« L'Enfant et le Maître d'école » ; " Le Renard et la Cigogne ») ; Livre II (« L'Astrologue qui se laisse tomber dans un puits »; "Le Loup plaidant contre le Renard par-devant le Singe »; "Les deux Taureaux et une Grenouille »); Livre IV (" Le Vieillard et ses Enfants "; "Le Renard et le Buste »; "Le Singe et le Dauphin »): Livre V («La Vieille et les deux Servantes »; «L'Aigle et le Hibou ») ; Livre VII (« La Laitière et le Pot au lait "; " Le Chat, la Belette et le petit Lapin ») ; Livre VIII («Les deux Amis » ; "L'Éducation ").

(33) A.-M. Bassy, Les Fables de La Fontaine. Quatre siècles d'illustration, op. cit., p. 74.

(34) Ibid., p. 85.

(35) Ibid., p. 119.

(36) Ce thème s'impose après 1750 selon F. Pupil, op. cit., p. 355-356.

(37) Outre la fable mettant en scène César et Laridon ("L'Éducation », livre VIII), Percier a sélectionné « Le Singe et le Dauphin » (livre IV), texte qui engage la réflexion sur l'altérité à travers l'idée de prudence. Voir notre article : "Les Fables pédagogiques : périégèse éducative et déambulation poćtique ", dans Céline Bohnert, Stéphane Macé, Bernard Teyssandier, Jean de La Fontaine, Le Laboratoire des Fables, Paris, PUF, 2011, p. 70-101, notamment p. 96-98.

(38) A. Chastel, L'Art français, op. cit., p. 11. 


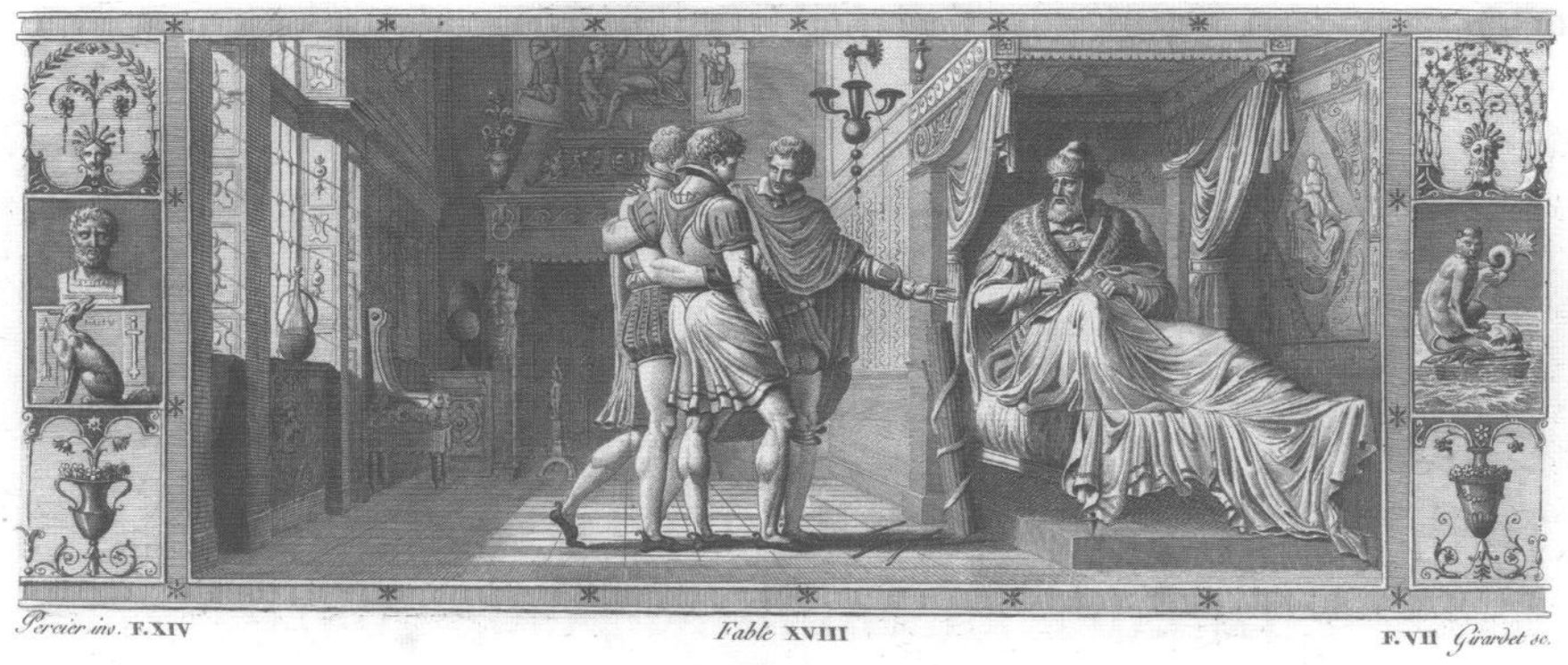

Fig. 1 : Bandeau du Livre IV : « Le Vieillard et ses enfants" (panneau central).

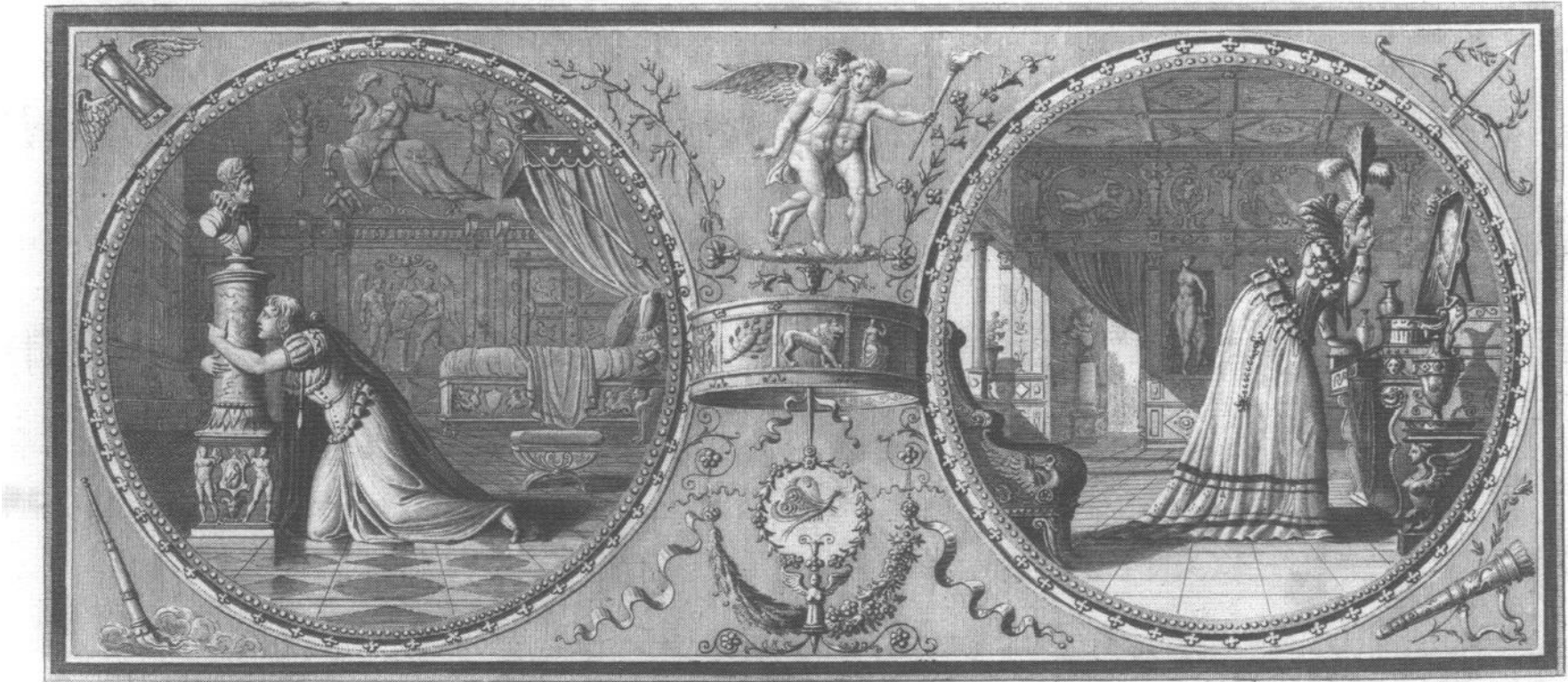

Tercier inv.

Fable XXI.

Girardet ise.

Fig. 2 : Bandeau du Livre VI : « La Jeune Veuve». 


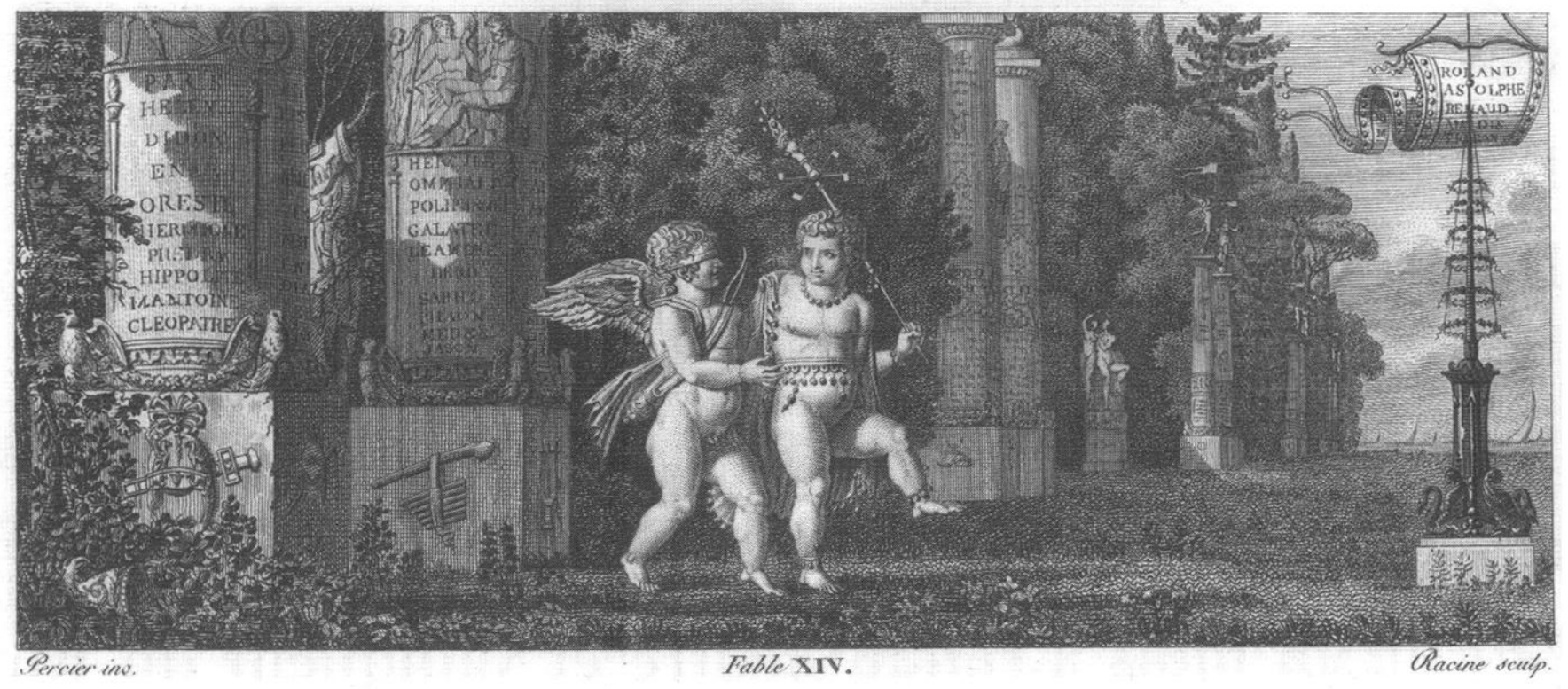

Fig. 3 : Bandeau du Livre XII : « L'Amour et la Folie».

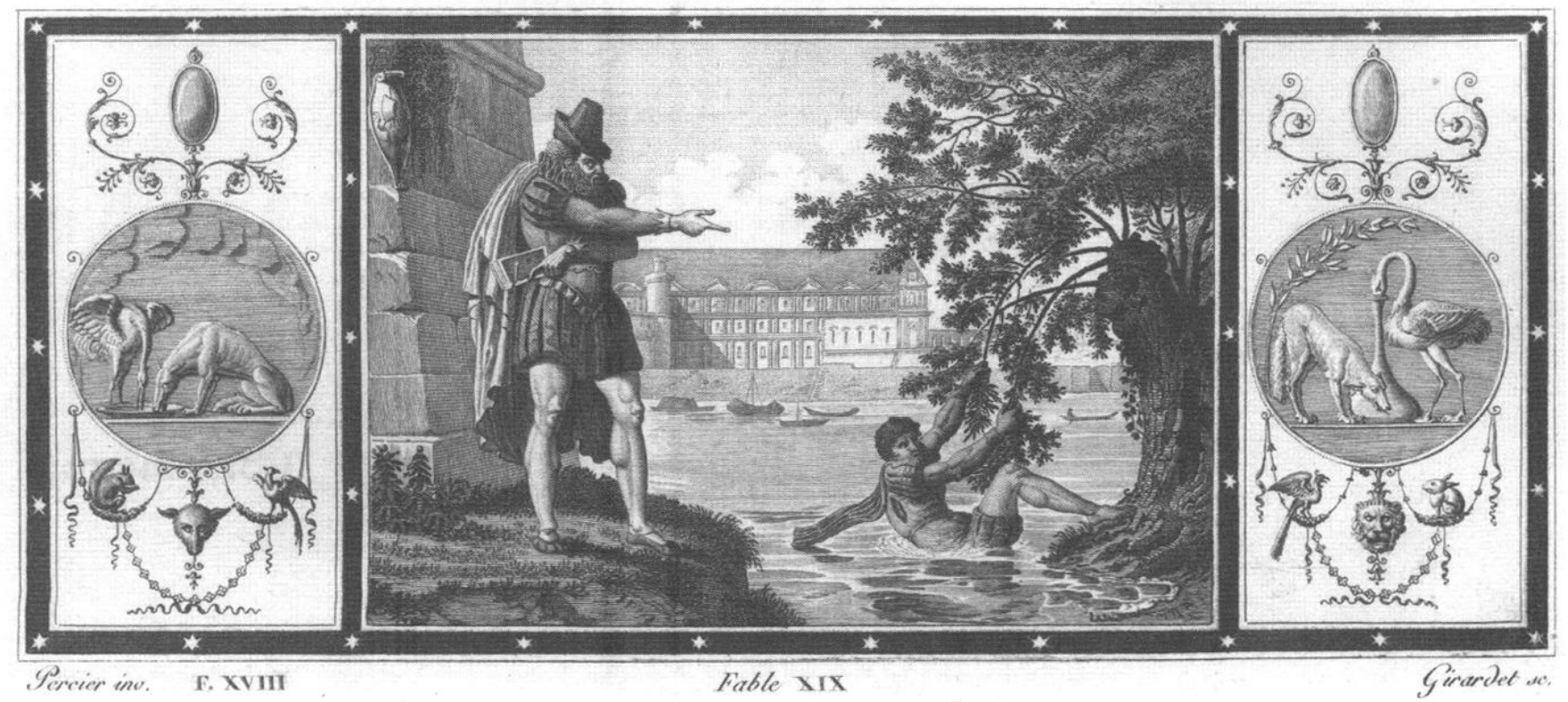

Fig. 4 : Bandeau du Livre I : «L'Enfant et le maître d'école ». 
qui est illustrée que les vertus de la leçon qu'elle est supposément capable d'inspirer (figure 3). Alors que le poète se refuse, pour le coup, à moraliser,

Mon but est seulement de dire, à ma manière, Comment l'aveugle que voici

(C'est un dieu), comment, dis-je, il perdit la lumière,

Quelle suite eut ce mal, qui peut-être est un bien ;

J'en fais juge un amant, et ne décide rien ${ }^{39}$.

Percier donne une dimension normative et prescriptive à la fable. Sa gravure, pour gracieuse qu'elle soit, n'en reste pas moins explicite dans ses intentions édifiantes : le drapeau qui claque au vent (marque habituelle de vanité) et les inscriptions gravées sur les colonnes désignent à la postérité des couples à la réputation ternie ; un bas-relief illustre d'ailleurs l'épisode emblématique d'Hercule chez Omphale ; quant aux voiles flottant sur l'eau, elles accompagnent symboliquement la condamnation de l'amour vénal, source d'infamie et d'inconstance.

Le phénomène de distorsion entre le texte lafontainien et l'image est plus manifeste encore pour le bandeau du livre I illustrant la fable de " L'Enfant et le Maître d'école » (figure 4). Alors que la vignette de Chauveau, en parfaite conformité avec le texte de La Fontaine, figure le maître sous les traits d'un pédant coiffé d'un bonnet de docteur, l'image de Percier évoque la vitalité et la virilité de la leçon. Le Barbe Bleue qui tance le freluquet récalcitrant n'est plus un barbacole, et le fait qu'il soit vêtu à la mode Henri III ${ }^{40}$ ne suffit pas à le rendre ridicule.

En 1802, Didot et Percier s'ingénient ainsi à héroïser la fable, témoignant d'une manière de lire l'œuvre lafontainienne et sans doute aussi de la faire lire. Mais plutôt que d'effectuer une greffe poétique plus ou moins improbable à la manière du père Audin ${ }^{41}$, ils travaillent la représentation du genre dans le but d'affirmer son autorité et même sa majesté. Le premier typographiquement, le second plastiquement : avec Didot l'apologue subit un traitement épigraphique ${ }^{42}$, avec Percier, il devient une curiosité esthétique. Mais la logiquc qui préside à cette lecture régénérée d'un « petit genre » $\mathrm{s}$ 'apparente aussi à un coup de force : l' « humeur franche et libre ${ }^{43}$ » de la fable est subordonnée à un principe supérieur où prévalent l'ordre, la symétrie, la netteté et l'immutabilité. La rêverie architecturale $e^{44}$ qui préside à la réalisation de cette édition contribue donc aussi à transformer l'œuvre qu'elle s'approprie, ou qu'elle tente peut-être imprudemment de s'approprier. Les espaces marmoréens de l'édition du Louvre ne sont plus tout à fait ceux d'un livre en effet, ils évoquent d'autres formes prépondérantes, celle du musée, celle du mausolée aussi. Non pas que Percier et Didot aient cherché à ensevelir les Fables en leur construisant un vaste et somptueux tombeau - " calme bloc ici-bas $\mathrm{chu}^{45} \gg$. Mais à trop vouloir honorer la poésie, l'un et l'autre figent les « légères peinture ${ }^{46}$ » de La Fontaine dans un rituel typographique et iconographique dont le degré d'impeccabilité n'est pas sans évoquer, en définitive, le cérémonial impérieux et compassé de grandes et froides funérailles.

Bernard TEYSSANDIER Université de Reims Champagne-Ardenne
(39) Fables, XII, 14

(40) « Il cst singulier qu'un style faisant référence constante au passé monarchique ait survécu au choc des événements révolutionnaires. [...] Avec l'ère des musćes, une sorte d'esprit archéologique s'empara des illustrateurs : les cuvres traduisirent parfois naïvement leur admiration pour les monuments redécouverts ", F. Pupil, Le Sivle troubadour ou la nostalgie du bon vieux temps, op. cit., p. 326 et 329.

(41) Audin, Fables héroüques, comprenant les véritables maximes de la politique chrétienne et de la morale. avec des discours enrichis de plusieurs histoires, tant anciennes que modernes, (gravures de François Chauveau), Paris, Jean Gaillard, 1648. Dans l'« Apologie en faveur des fables » qui ouvre la première partie de son recueil, Audin repousse l'idée d'une puérilité de la fable mais plaide en revanche pour une dimension politique de l'apologuc : « Sous le nom de fables, je n'entends pas d'autorité les contes ni les sornettes des vieilles, mais bien celles qui, pour être artistement et judicieusement inventées, confirment les sages dans la recherche de la vertu, font abhorrer le vice aux méchants, et donnent de grandes lumières à ceux qui sont les chefs et les arbitres de la vie civile [...]. Les rois mêmes n'ont pas dédaigné de parler ce langage. [...] Certes, quoique les autorités que je rapporte soient très considérables, les ignorants ne laisseront pas de croire que l'incomparable Ésope n'a inventé les fables que pour faire taire les enfants quand ils pleurent ou pour les endormir plus facilement quand on les berce. Et néanmoins la vraie politique s'y voit honorablement établic, la façon de bien vivre s'y trouve dépeinte avec toutes ses couleurs : la charge du prince, le devoir du peuple, le bonheur de la république s'y font remarquer avec respect $[\ldots]$. JésusChrist, même, lorsqu'il conversait parmi les hommes ne parlait le plus que par des paraboles, c'est-à-dire par fables inventées très sagement pour vaincre un peuple infidèle. [...] Comme tout cela est plein de mystères et que Jésus-Christ est la Vérité même, il faut tenir que les fables dont il se servait étaient le caractère de la vérité et qu'elle ne pouvait être ni plus noblement ni plus utilement représentée. [...] Quoique la fable semble ridicule pour introduire des animaux et des arbres qui parlent, elle n'en est pas moins profitable : au contraire, elle est d'autant plus à rechercher que le suc qu'elle enferme artistement est le vrai nectar et l'ambroisie des dieux ou pour parler plus intelligiblement la pâture des sages d'autant plus salutaire au cœur qu'elle est désagréable à l'oreille " (texte non paginé). Voir notre article, "Ésope, quel modèle pour le prince ? ", Le Fablier, n" 20, 2009 , p. $41-46$ et p. $50-51$.

(42) "Pour juger de la valeur des innovations du citoyen Didot, il faut se demander quel est le but de l'imprimerie. Est-ce la beauté d'un tableau, ou la facilité d'une lecture ? Est-ce la délicatesse des formes des lettres ; ou l'évidence des formes des lettres ? ", Discours sur l'art de l'imprimerie, texte cité dans Les Didot. Trois siècles de typographie, op. cit., p. 29.

(43) J. de La Fontaine, "Le Renard anglais ", Fables, XII, 23.

(44) Sur la " poesie enchanteresse de l'architecture ", voir par exemple Étienne-Louis Boullée, LArchitecture visionnaire et néo-classique, textes réunis par Jean-Maric Pérouse de Montclos, Paris, Hermann, 1993, p. 34 sqq.

(45) Mallarmé, "Le tombeau d'Edgar Poe ", v. 12

(46) «À Monseigneur le Dauphin ». épitre en vers précédant le livre I des Fables choisies mises en vers (1668), v. 14. 